\title{
Overview:
}

The COVID-19 pandemic and responses to help prevent its spread have had far-reaching impact on all Americans, and particularly on cancer patients and survivors. Risk of infection, overloaded health care systems, shortages of food and supplies, and economic challenges all create unique barriers to achieving and maintaining health while fighting cancer and after.

To better understand the impact of COVID-19 on the vulnerable population of US cancer patients and survivors, the American Cancer Society Cancer Action Network (ACS CAN) initiated a survey on March 25,2020 . The survey was deployed across a two week period to the 3,055 cancer patients and survivors participating in the Survivor Views initiative, and was also shared through ACS CAN social media and email channels to recruit additional respondents.

The survey focused on respondents' experiences accessing health care as a result of the COVID-19 pandemic, including availability of appointments and services, ability to pay for care, and concerns about being able to safely obtain needed health care treatments and services in the future. The webbased survey was taken by more than 1,200 cancer patients and survivors. This sample provides a margin of error $+/-3 \%$ and $96 \%$ confidence level.

The research provides important insights into the experiences of cancer patients and survivors in safely accessing and affording health care during the COVID-19 pandemic.

\section{Key Findings:}

Key findings from the survey include:

- Fifty percent of cancer patients and survivors reported some impact to their health care due to the COVID-19 pandemic. Twenty-seven percent of patients who are currently in active treatment report a delay to their treatment, and more than 1 in $8(13 \%)$ of those in active treatment have had care delayed without knowledge of when it will be rescheduled.

- The economic impact of the pandemic is affecting many cancer patients and survivors, with $38 \%$ of respondents reporting a notable impact on their financial situation that affects their ability to pay for care. Nearly half of cancer patients and survivors whose annual household income is $\$ 30,000$ or less are worried that the financial impact of the COVID-19 pandemic will make it hard for them to afford the care they need.

- A third of cancer patients and survivors are worried about the impact of COVID-19 on their ability to get treatment for their cancer, a concern that is particularly acute for those currently in active treatment ( $40 \%$ of whom express worry).

\section{Detailed Survey Findings}

The survey included a wide range of US cancer patients and survivors who have been diagnosed with and/or treated for cancer within the last five years. Fifty-one percent of respondents are currently being treated for cancer. Among respondents who are not currently in active treatment, more than half $(60 \%)$ received their last treatment within the last year. 


\section{COVID-19 Pandemic Impact on Cancer Patients and \\ Survivors \\ Survey Findings Summary}

Nearly half of respondents (49\%) receive insurance coverage through an employer, and $32 \%$ are covered by Medicare. Seven percent have privately purchased health care, such as through an exchange, and four percent are covered by Medicaid. Compared to insurance coverage for the US general population, the survey included more respondents on Medicare and fewer on privately purchased plans or Medicaid ${ }^{1}$, which is likely a result of increased cancer incidence rates among older Americans.

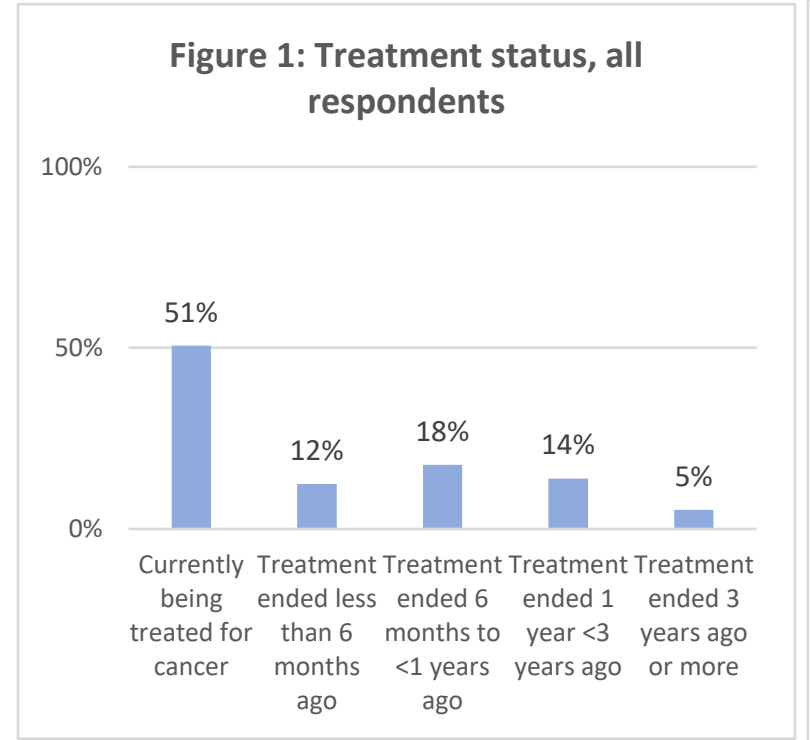

Figure 2: Insurance coverage, all respondents

Other or no

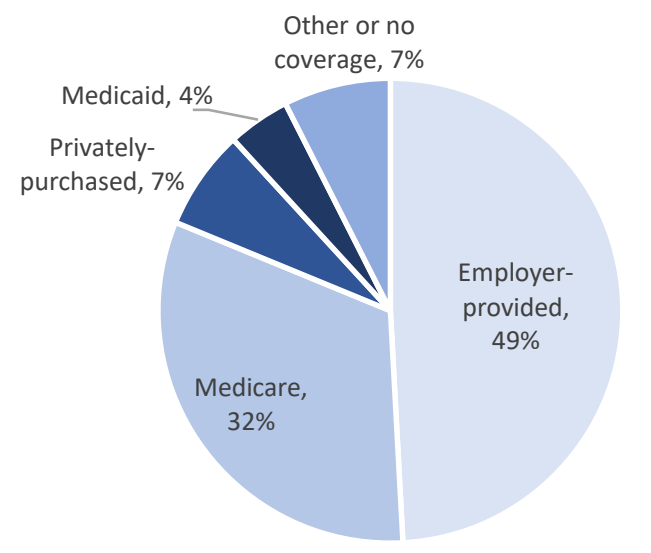

Fifty percent of all respondents to the survey report some change, delay, or disruption to their health care as a result of the COVID-19 pandemic. Among patients and survivors currently in active treatment, the incident of changes, delays, or disruptions increases to $55 \%$. The most commonly experienced issue is a delay to care or treatment ( $24 \%$ of all respondents, $27 \%$ of respondents in active treatment). One in $8(12 \%)$ respondents and $13 \%$ of those in active treatment report that they have had care or treatment delayed and do not know when it will be rescheduled, indicating a great deal of uncertainty about their future ability to access care.

In addition to delays and cancellations, many cancer patients and survivors reported the transition of health care services from in-person to remote delivery. Fifteen percent of respondents have had an inperson provider visit changed to a phone or video visit.

\footnotetext{
${ }^{1}$ For 2018 US insurance coverage sources, please see: https://www.kff.org/other/state-indicator/total-population/
} 


\section{COVID-19 Pandemic Impact on Cancer Patients and \\ Survivors \\ Survey Findings Summary}

Figure 3: Most common affects of COVID-19 on health care, all respondents

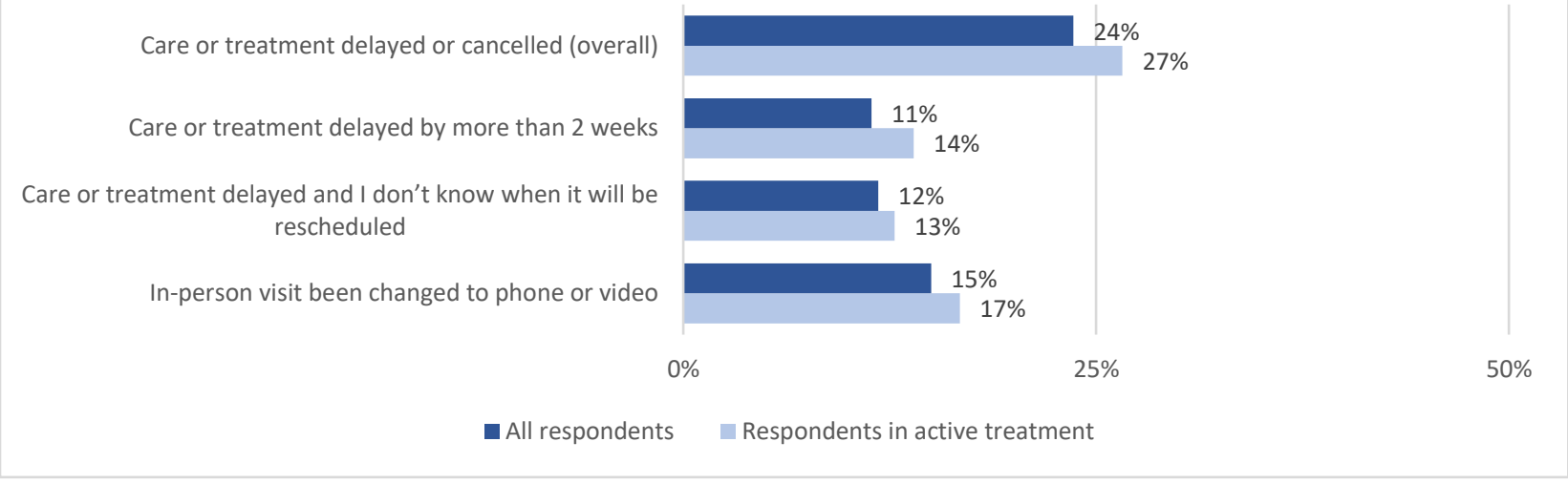

Among respondents whose care has been cancelled, delayed, or changed by the pandemic, the most commonly impacted services are in-person provider visits ( $50 \%$ of impacted respondents), supportive services such as therapy (20\%) and imaging procedures to monitor the growth of their cancer (20\% of impacted respondents). Eight percent of respondents who are currently in active treatment report that their anti-cancer therapy (e.g., chemotherapy, immunotherapy) has been impacted by the COVID-19 pandemic.

Beyond the already observed impact of COVID-19 on cancer patients' and survivors' access to health care, respondents also expressed concern about their future ability to access treatment for their cancer. One in three (34\%) respondents agreed or strongly agreed with the statement "I am worried that the COVID-19 outbreak and the response to it will make it hard for me to get treatment for my cancer." This concern is even greater among those in active treatment, $40 \%$ of whom agreed or strongly agreed with the statement.

The COVID-19 pandemic and resulting slowing of economic activity has had substantial impact on the ability of cancer patients and survivors to afford care. Nearly four in ten (38\%) respondents report COVID-19 has had a notable impact on their financial situation that affects their ability to pay for health care. The most common financial issues affecting access to care for cancer patients and survivors include reduced work hours (14\%), reduced investment values (11\%), difficulty affording food and supplies in order to stay at home to avoid contracting the virus (9\%) and loss of a job (8\%).

Reduced work hours and lost jobs are of particular concern with regard to paying for health care because these have the potential to impact access to health insurance. Forty-three percent of respondents who reported that they or a family member living with them had lost a job had employersponsored health insurance. Among those who indicated they or a family member had work hours reduced, $58 \%$ had employer-sponsored coverage.

Twenty-eight percent of all respondents expressed worry about the financial impact of COVID-19 making it difficult to pay for the health care they need as cancer survivors. This concern was highly correlated with income, with $46 \%$ of those earning $\$ 30,000$ or less reporting they are worried. Even in households 


\section{COVID-19 Pandemic Impact on Cancer Patients and}

Survivors

Survey Findings Summary

earning more than $\$ 110,000^{2}$, one in five (21\%) respondents expressed concern about financial impact. This difference is statistically significant at the $<.001$ level.

Figure 4: Agree/Strongly agree with the statement"I am worried that the financial impact of COVID-19 will make it hard for me to afford the care I need as a cancer survivor", by household income

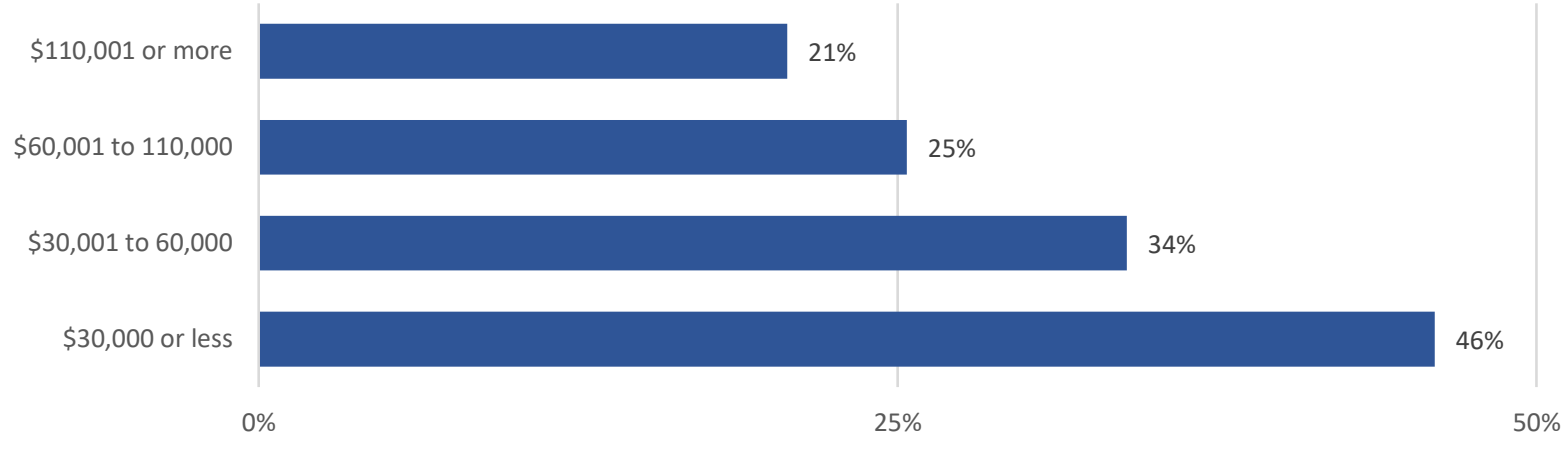

\section{Methodology}

ACS CAN's COVID-19 survey was administered to participants in our Survivor Views research panel. Survivor Views is an ACS CAN initiative designed to enhance the organization's mission to end suffering and death from cancer. Data provided by cancer patients and survivors as part of this project allows for a greater understanding of their experiences and opinions on cancer-related issues and gives voice to cancer patients and survivors in the shaping and advocating of public policies that help prevent, detect, and treat cancer and promote a more positive quality of life for those impacted.

Due to the urgent need to understand the impact of COVID-19 on cancer patients and survivors, this survey was simultaneously deployed outside of the Survivor Views panel through an anonymous survey. The anonymous survey link was distributed via email to ACS CAN volunteers and promoted through ACS CAN social media accounts.

To ensure the protection of all participants in this survey, all research protocols, questionnaires, and communications were reviewed by the Morehouse School of Medicine Institutional Review Board.

The survey population is comprised of individuals who meet the following criteria:

- Diagnosed with and/or treated for cancer within the last five years

- Over the age of 18

- Reside in the US or US territories

\footnotetext{
${ }^{2}$ Note that household income information was provided optionally by Survivor Views participants and was therefore only available for 797 respondents.
} 


\section{COVID-19 Pandemic Impact on Cancer Patients and}

\section{Survivors}

\section{Survey Findings Summary}

This survey was conducted using a web-based instrument sent to 3,055 Survivor Views cohort members and promoted to non-panelist respondents through email and social media promotion. The data were collected between March 25 and April 8, 2020. A total of 1,219 participants responded to the survey, providing a margin of error $+/-3 \%$ and $96 \%$ confidence level.

\section{About ACS CAN}

The American Cancer Society Cancer Action Network (ACS CAN) is making cancer a top priority for public officials and candidates at the federal, state and local levels. ACS CAN empowers advocates across the country to make their voices heard and influence evidence-based public policy change as well as legislative and regulatory solutions that will reduce the cancer burden. As the American Cancer Society's nonprofit, nonpartisan advocacy affiliate, ACS CAN is critical to the fight for a world without cancer. For more information, visit www.fightcancer.org. 